\title{
Accident and emergency department led implementation of ketamine sedation in paediatric practice and parental response
}

\author{
V J Holloway, H M Husain, J P Saetta, V Gautam
}

\begin{abstract}
Objective-To evaluate accident and emergency (A\&E) department led practice of ketamine sedation for painful, short procedures in the paediatric population and to ascertain parental response.

Methods-Analysis of retrospective data for all children who received ketamine sedation over a 20 month period in a district general hospital. A data extraction form was used to record age, sex, dose, indication, side effects, and outcome. The parents were contacted by telephone afterwards and asked standardised questions about the child's treatment, their progress after discharge, and overall satisfaction with the treatment.

Results-Intramuscular ketamine was administered to 100 children under 12 years of age during the study period. The drug caused no adverse events pre-operatively or intraoperatively. The main early postoperative complication was vomiting $(14 \%)$. Ninety three per cent of patients were discharged the same day. No reattendance or treatment attributable to ketamine related side effects were necessary. Over the 24 hours after discharge, vomiting occurred in $12 \%$ and ataxia in $15 \%$ of patients. Ninety nine per cent of parents were either very satisfied or satisfied with ketamine sedation and were willing for their child to receive it again, if required.

Conclusion-This study, while confirming the known safety of ketamine sedation, established its suitability for "independent" use within $A \& E$ departments by suitably qualified staff.

(F Accid Emerg Med 2000;17:25-28)
\end{abstract}

Keywords: ketamine; children; sedation; suturing

Painful but brief clinical interventions, for example, wound exploration, suturing and incision and drainage, are commonly required for children attending accident and emergency (A\&E) departments. Most of these procedures are relatively short lived and achieved with local anaesthetic or restraint alone. However, the situation arises when a still, cooperative child is essential. Many methods of sedation and analgesia have been described for children in these circumstances; local anaesthetic infiltration alone, administration of benzodiazepines or opioids by varied routes, inhalational analgesia and general anaesthetic. ${ }^{1-4}$
There are drawbacks to all these options and inadequate sedation or anxiolysis may well occur with some of these techniques. While restraint alone achieves the desired end result, in some patients this may be at the expense of losing the child's or parents' confidence, or both.

General anaesthetic given by an experienced anaesthetist may be seen as the gold standard to aim for, but the reality for most $A \& E$ departments is that the prevailing anaesthetic and surgical service would not be able to cope with the demand. Recent developments in A\&E department organisation have made exploring alternative methods of safely sedating children possible. We would not however advocate that ketamine is used in the place of a general anaesthetic when dealing with complex wounds that obviously need formal explorations in an operating theatre.

The ideal is to treat children with minimal distress, disruption, delay, and avoidance of hospital admission. Ketamine administration within the A\&E department offers a solution to several of these issues and may also have a favourable impact on scarce hospital resources. In view of these clinical advantages, this study was conducted to evaluate the success of providing ketamine sedation within the $A \& E$ department of a district general hospital in the United Kingdom by senior A\&E department medical staff.

\section{Methods}

The A\&E department records of all children given ketamine $(n=100)$ in our department over the previous 20 months (February 1997October 1998) were analysed. Information was taken from notes made in the $\mathrm{A} \& \mathrm{E}$ department records at the time (a proforma had not been introduced at that stage). Criteria for use of ketamine included situations where it was essential for the child to remain still (for example, lip lacerations crossing the vermilion border and other facial wounds), where attempts to use local anaesthetic alone had failed or likely to fail, and where the parents were unwilling for their child to be restrained. Wounds considered were those deemed suitable for primary closure/repair or where exploration was needed for removal of foreign bodies in the A\&E department. All parents gave written, informed consent after a choice of treatment options had been discussed. The clinicians administering ketamine were all experienced emergency medicine consultants, specialist registrars, or staff grades who had a 
Table 1 Telephone survey: questions and responses $(n=61)$

1 Did your child have any of the following during the 24 hours after discharge?:
$\begin{array}{ll}\text { (a) Vomiting } & 12(19.6 \%) \\ \text { (b) Loss of coordination } & 15(24.5 \%) \\ \text { (c) Nightmares } & 2(3 \%) \\ \text { (d) Drowsiness } & 7(11 \%) \\ \text { (e) Unusual behaviour } & 4(6 \%) \\ 2 \text { Would you be willing for your child to have ketamine again if necessary? } & 60(98.4 \%) \\ \text { Yes } & 1(1.6 \%) \\ \text { No } & 52(85 \%) \\ 3 \text { Overall, how satisfied were you with this method of sedation? } \\ \text { Very satisfied } & 9(14 \%) \\ \text { Satisfied } & 1(1 \%) \\ \text { Dissatisfied } & \end{array}$

^This child was agitated and vomited during recovery, which caused distress to the mother.

minimum training of an Advanced Paediatric Life Support course and broad paediatric experience. Two doctors and a nurse were always present during the procedure and all surgery was carried out by the A\&E department clinician. Every child was given supplemental oxygen by facemask and was closely observed by the nurse until recovery, who monitored vital signs and pulse oximeter readings. (See protocol for use of ketamine, table 4.)

Parents of this patient group were also contacted by telephone. To avoid interobserver bias, the same person (VJH) interviewed the parents using a standardised questionnaire with a pre-determined choice of answers. (This survey was undertaken from between one week to 14 months after procedure averaging approximately five months after the event.) The opportunity for respondents to add any other comments was given. Details of non-responders were re-checked and all homes were called on a minimum of three occasions, at different times, to elicit a response. Table 1 lists the questions asked in the telephone survey.

Descriptive statistical methods were applied to both sets of data.

\section{Results}

One hundred children (male 56, female 44) received ketamine over a consecutive 20 month period (fig 1). The mean dose given was 5.54 $\mathrm{mg} / \mathrm{kg}$ (3.65-8.91 mg/kg); mode $5.0 \mathrm{mg} / \mathrm{kg}$. (When ketamine was initially introduced
Table 2 Procedures undertaken

\begin{tabular}{ll}
\hline Sutures/repair/exploration & \\
Lip lacerations & 18 \\
Facial wounds & 25 \\
Hand and fingertip injuries & 25 \\
Other & 10 \\
Subtotal & 78 \\
Removal of foreign body & 9 \\
Incision and drainage & 7 \\
Fracture manipulation & 3 \\
Lump excision & 1 \\
Dressing change & 1 \\
Knee aspiration & 1 \\
Total & 100 \\
\end{tabular}

higher doses were used on the first patients. Two children were given a low dose $<5 \mathrm{mg} / \mathrm{kg}$; it was not clear from the notes why this was.)

Five children from the series required a second dose to achieve adequate sedation.

Thirty eight children were given atropine additionally, either orally $(30 \mu \mathrm{g} / \mathrm{kg})$ or intramuscularly (10-20 $\mu \mathrm{g} / \mathrm{kg}$ ). Atropine was introduced in the latter half of the study to prevent ketamine associated hypersalivation. This was after a suggestion made by our anaesthetic colleagues, although hypersalivation had not been a problem in our experience before this.

Pulse and blood pressure changes from baseline to maximum readings during the procedure were analysed but found to be statistically insignificant in all children whether given atropine or not. Procedures undertaken in table 2 were all injuries suitable for $A \& E$ department management but necessitated a still, cooperative child. Lip injuries involved the vermilion border, facial wounds were significant enough to require several sutures rather than just glue or steristrips, and hand wounds were mainly finger tip injuries that involved the nail bed or required suturing, or both.

\section{Results}

No child needed admission because of side effects of ketamine and there were no reattendances recorded attributable to ketamine effects (table 3 ). There were no complications from the surgery itself. The majority of patients (93) were discharged home that day. One child was transferred to the plastic surgeons after exploration of a wound in the palm revealed a

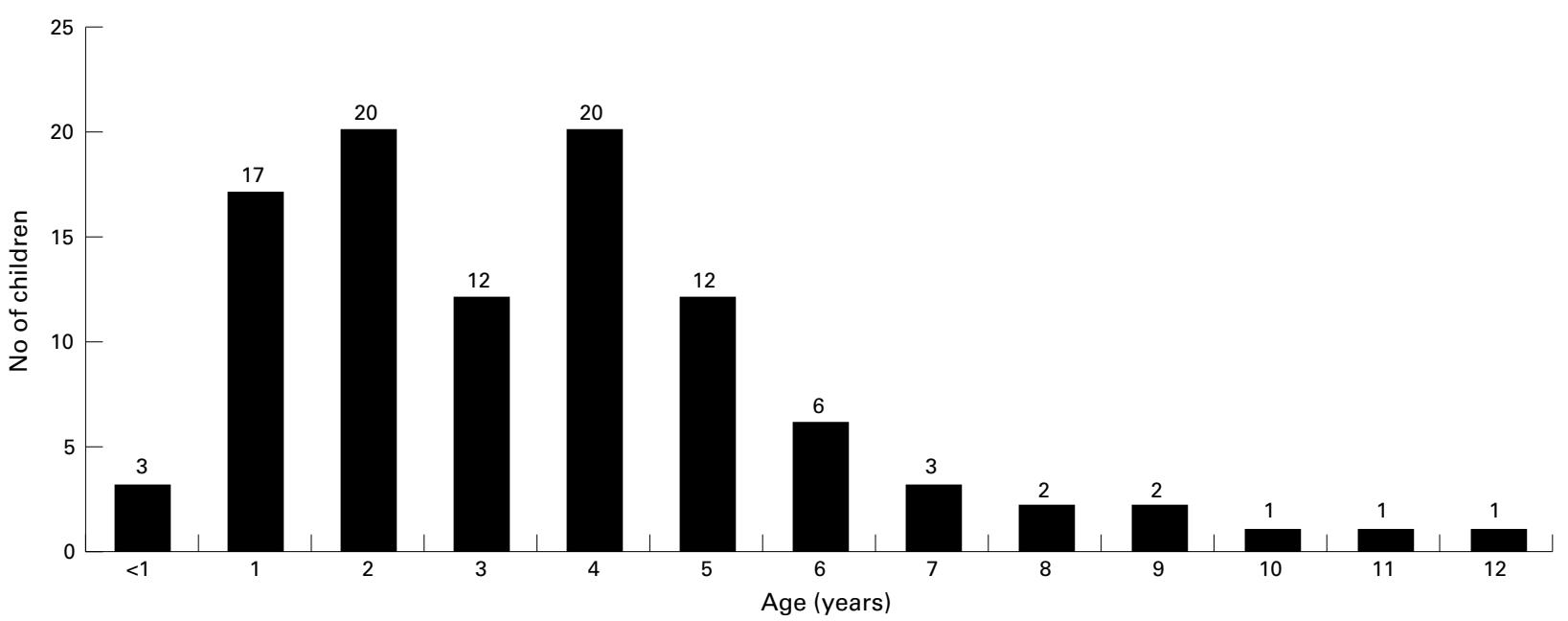

Figure 1 Age distribution of the group of patients $(n=100)$ receiving ketamine during the study period (20 months). 
Table 3 Complications/side effects

\begin{tabular}{lllll}
\hline & Procedure & Recovery & Post-discharge & $>24$ hours \\
\hline Airway problems & 0 & 0 & 0 & N/A \\
Vomiting & 0 & 14 & 12 & 0 \\
Ataxia & 0 & 0 & 15 & 0 \\
Agitation/nightmares & 0 & 6 & 2 & 0 \\
Unusual behaviour & 0 & 0 & 4 & 0 \\
\hline
\end{tabular}

Procedure $=$ administration of ketamine to completion of intervention. Recovery $=$ two hours after discharge. Post-discharge $=$ up to 24 hours after leaving hospital. N/A $=$ not applicable.

flexor tendon injury. Six children were admitted to the ward (parental anxiety: 2, treatment of femoral fracture: 1 , child deeply asleep: 1 , further investigation: 1 , unknown: 1 ).

Table 4 shows our suggested, adapted, protocol for independent use of ketamine sedation.

\section{Discussion}

The fundamental principle of treating children with minor injuries requiring short, painful interventions, is that no child should be subjected to unnecessary discomfort or distress. Treatment of most of these injuries can be undertaken safely within the setting of an $\mathrm{A} \& \mathrm{E}$ department. Ketamine sedation is an option that has found favour in the USA and developing worlds, ${ }^{5-8}$ but has yet to gain full acceptance in the United Kingdom.

Ketamine does not fit easily into standard drug classifications. At low doses full general anaesthesia is not achieved, rather a dissociated state in which airway and respiratory tone are maintained. Concern may be expressed regarding the potential risk of cardiorespiratory complications. This issue should be considered within the context of regular administration of sedative drugs within $\mathrm{A} \& \mathrm{E}$ departments for various procedures, adverse effects from these are considered manageable within departments. Hazards of sedation are well known and written guidelines, already followed. ${ }^{10-12}$ It would seem logical for these guidelines to be applied to ketamine sedation. While the absolute risk of complications in the paediatric population is not quantified, this drug is unlikely to be more unsafe than other commonly used sedatives. The specific dangers of airway compromise or cardiorespiratory instability are in fact suggested to be less with ketamine. ${ }^{13-15}$

The size and staffing levels in A\&E departments using the drug should be such that there is substantial skill available to manage complications should they arise. Routine emergency backup in all hospitals receiving acute admissions is also generally available from anaesthetic and paediatric staff by prior arrangement.

Advantages of ketamine sedation within the A\&E department are many: it prevents excessive distress for the child, avoids bed occupancy hospital admissions and can offer economy of time and resources for surgeons, anaesthetists, paediatricians, theatre and ward staff. Using ketamine does occupy a senior $A \& E$ department clinician and nurse for the duration of the procedure and while the child is recovering. In our experience, this does not take much longer than completing the procedure under local anaesthetic alone, after the time taken to cajole and restrain the child and pacify the parents. Procedures themselves are also made far easier and quicker as the child remains still and enables more thorough wound exploration/closure. (Unfortunately

Table 4 Protocol for administration of ketamine to children in the A\&E department

\section{Patients:}

Children requiring short, painful interventions. For example, suturing complex lacerations, incision and drainage of abscesses, minor orthopaedic manipulations, repair of finger tip injuries, etc.

Contraindications:

- Active lung disease or infection including URTI. (Asthma not contraindicated.)

- Cardiovascular disease, for example, congenital, cardiomyopathy, hypertension.

- Head injury if associated with history of loss of consciousness, vomiting, altered mental state, or any sign of raised intracerebral pressure.

- Central nervous system disease, for example, hydrocephalus, intracranial mass, epilepsy.

- Others: porphyria, thyroid disease, glaucoma, psychosis.

Preparation:

- Ensure child is starved for minimum of three hours.

- Record weight in kilograms.

- Obtain written consent from guardian after explaining potential risks/side effects of procedure.

- Apply EMLA cream 60 minutes before procedure over the area for injection.

- Encourage parent to stay with child during procedure and recovery.

Requirements:

- Presence of two doctors, at least one trained in paediatric resuscitation.

- Presence of qualified nurse ideally with paediatric experience to monitor the child until fully recovered.

- Record baseline vital signs before drug administration.

- Equipment needed: oxygen, suction, vital sign monitors including pulse oxymeter and a full paediatric resuscitation trolley. (Intravenous access not essential.)

- A designated area where noise and disturbance are minimal.

Dose:

Ketamine $5 \mathrm{mg} / \mathrm{kg}$ as initial dose $2 \mathrm{mg} / \mathrm{kg}$ if repeat dose required at 10 minute intervals. Atropine (100-200 $\mu \mathrm{g} / \mathrm{kg}) \mathrm{may} \mathrm{be} \mathrm{added} \mathrm{to} \mathrm{minimise} \mathrm{ketamine} \mathrm{associated}$ hypersecretion.

Procedure:

- Ketamine (and atropine if required) are administered intramuscularly into the pre-anaesthetised skin over buttock or thigh. Child to be held in parent's arms if possible.

- Once sedated the child is placed on their side on a tilting trolley with continuous pulse and oxygen saturation monitoring.

- Optimise airway position, occasional gentle suction of oropharynx may be required to clear secretions.

- Monitor continuously until fully recovered.

- Recovery area should be quiet if possible, with minimal tactile stimulation. Mean recovery time is $60-140$ minutes.

Side effects:

Observe for: airway compromise - that is, laryngospasm, apnoea, hypersalivation.

Also, vomiting, agitation, hallucinations, nightmares, ataxia.

Discharge:

- When child has returned to pre-treatment level of awareness, recognition, speech, and purposeful activity.

- Instruct parents to give nothing by mouth until fully orientated and closely observe ambulation for next 24 hours. 
data were insufficient to give exact times of clinician involvement.) The use of ketamine is also positive in terms of job satisfaction; clinicians are able to treat the child from start to finish without the need to refer on relatively minor wounds for closure, simply because of the need for sedation.

$\mathrm{A} \& \mathrm{E}$ departments considering using ketamine in children need to make provision for training of nursing staff and locate a suitable, quiet environment for recovery of the child. Staffing levels need to be adequate.

A pleasing outcome from this study was the parents' response. On telephone questioning, many had had previous experiences in an $A \& E$ department where their child was restrained and they were glad to have been offered a more humane alternative method of treatment. The majority of parents in this study stayed with their child throughout the procedure and had seen treatment from start to finish; all but one of the parents were willing for their child to undergo ketamine sedation again, should the need arise. We recognise the fact that this was a retrospective study, and in some instances there was a long time gap between the event and questioning; because of this there may be limitations in recollection of events however, all parents questioned remembered the event surprisingly vividly, presumably because any injury to their child requiring hospital treatment is very distressing and remains clearly in the memory. The main side effect encountered was postoperative vomiting, on the whole this was transient and a minor inconvenience. The ataxia noted in 15 children after discharge was short lived, with parents reporting that their child had been unsteady on their feet until bedtime but had awoken the next morning free of symptoms. Agitation during recovery was noted in six children, in five patients this took the form of a type of hallucination where the children looked as if they were reaching out to touch or speak to imaginary objects. Only one child experienced what looked like unpleasant hallucinations/nightmares during recovery. Four children experienced unusual behaviour after discharge; this ranged from "being a bit naughty" to an English child speaking in an Irish accent (Irish recovery nurse?).

Local anaesthetic infiltration at the site of injury while under sedation may be used as an adjunct to analgesia so that the child awakes pain free.

Because of recent changes in emergency medicine training, speciality recognition and expansion of senior staff numbers, A\&E department doctors are increasingly able to maintain autonomy in their clinical practice.
The safety data from previous studies, our own experience (SAEM/FAEM Conference, Oxford, 1998, poster paper presentation) and comparisons with established practice show that the use of this drug is safe in the hands of non-anaesthetists. Since this study was completed we are aware that other departments have been using lower doses of ketamine, hence we have started a study using a smaller dose of ketamine with local anaesthetic augmentation and have introduced a proforma to collect data prospectively.

In the pressurised hospital environment of today the benefits of $\mathrm{A} \& \mathrm{E}$ department led sedation and treatment begin to add up.

The authors thank Mr David Gaunt FRCS, the clinical audit department at QE2 hospital especially Heather Davis, Carol Haynes and Ann Ainsworth.

Conflict of interest: none.

Funding: none.

Contributors

Dr V J Holloway, execution and coordination of the study, data collection and documentation. Designed and completed the telephone survey, discussed core ideas and issues. Main author of the paper. Mr H Husain, major contributor in data collection, documentation, analysis and interpretation. Discussed all core ideas and instigated much of the background research and literature scans. Mr J P Saetta, initiated and coordinated the original project, led discussion of core principles and ideas. Involved in the design and writing of the paper and was the moin cal analysis. Guarantors, J Saetta and V Holloway.

1 Ferguson S, Ball AJ. Sedation and sedative drugs in paediatrics. Br f Hosp Med 1996;55:611-15.

2 McGlone RG, Durham S. An alternative to 'Brutacaine': a comparison of low dose intramuscular ketamine with intranasal midazolam in children before suturing. 7 Accid Emerg Med 1998; 15:231-6.

3 Saccheti A, Schafermeyer R, Gerardi M. Paediatric analgesia and sedation. Ann Emerg Med 1994;23:237-49.

4 Proudfoot J, Petrack E. The six skills of highly effective paediatric sedation. Paediatric Emergency Medicine Reports 1997;2:79-90.

5 Green SM, Rothrock SG, Lynch EL, et al. Intramuscular ketamine sedation in the emergency department: safety profile in 1,022 cases. Ann Emerg Med 1998;31:688-97.

6 Green S. Ketamine sedation for paediatric procedures: part 1, a prospective series. Ann Emerg Med 1990;19:1024-32.

7 Green SM, Johnson NE. Ketamine sedation for paediatric Green SM, Johnson NE. Ketamine sedation for paediatric
procedures: part 2, review and implications. Ann Emerg procedures: part 2, revi

8 Green SM, Clem KJ, Rothrock SG. Ketamine safety profile in the developing world: survey of practitioners. Acad Emerg Med 1996;3:598-604.

9 White P, Way W. Ketamine - its pharmacology and therapeutic uses. Anaesthesiology 1982;56:119-36.

10 Reference withdrawn.

11 Green SM, Wittlake WA. Meeting the guidelines and standards for paediatric sedation and analgesia. Paediatric Emergency Medicine Reports 1997;2:67-78.

12 American College of Emergency Physicians. Clinical policy for procedural sedation and analgesia in the emergency fer procedural sedation and analgesia in the

13 Drummond GB. Comparison of sedation with midazolam and ketamine: effects on airway muscle activity. $\mathrm{Br} f$ Anaesth 1996;76:663-7.

14 Hazma J, Ecoffey C, Gross JB. Ventilatory response to $\mathrm{CO}_{2}$ following intravenous ketamine in children. Anaesthesiology 1989;70:422-5.

15 Petrack EM, Marx CM, Wright MS. Intramuscular ketamine is superior to meperidine, promethazine and chlorpromazine for paediatric emergency department sedation. Arch Pediatr Adolesc Med 1996;150:676-81. 